\title{
Finite quantum theory of fields and strings. 2. Gravitational self-regularization at Planck scales
}

\begin{abstract}
Zahid Zakir $^{1}$
Abstract

After the discovery of the slow growth of loop corrections with cutoff energy and the possibility of renormalization of the Lagrangians, the main unsolved problem of quantum field theory remained ultraviolet divergences, and the long search for a physical mechanism making regularization finite was unsuccessful. The main reason for this was the hasty conclusion that known phenomena were already taken into account and, as the result, the solution has been associated with radical hypotheses only. It is shown in the paper that the desired mechanism already existed initially and this is the gravitational time dilation. The standard treatment included high-energy quanta, but did not take into account their gravity, and in attempts to take into account it, gravity was considered only as one of the fields. But external gravitational field of quanta also slows down the local proper times in terms of the world time $t$ of distant observers. It is shown that at the Planck length, the gravitational radius of the Planck energy quanta, all processes freeze and therefore do not contribute to the $S$ matrix defined on the hypersurface of simultaneity $t=$ const. The freezing of quantum fluctuations means a strong redshift of high frequencies up to their vanishing and thus gravitation of quanta leads to their self-regularization. The nonlinearity of fields increases the gravitational effects, and hence the freezing, which even more reduces the high energy contributions. The ultraviolet finiteness of the loop corrections makes consistent non-renormalizable models too, if these corrections are small. For gauge fields and quantum gravity, the invariant Planck cutting of the integrals gives upper limits for the loop diagram corrections, which appear small and the perturbation theory series converge. The consequences of the finiteness of the Standard Model and quantum gravity are discussed.
\end{abstract}

Keywords: quantum fields, regularization, renormalization, quantum gravity

\section{Content}

Introduction .2

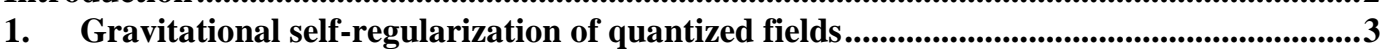

1.1. Gravitational freezing of fluctuations at Planck distances ................................................. 3

1.2. Gravitational self-regularization of loop diagrams ........................................................5

2. Finite Standard Model and Quantum Gravity ......................................................................7

2.1. The ultraviolet-finite quantum electrodynamics ............................................................... 7

2.2. Finiteness of other fields of the Standard Model ......................................................... 10

2.3. Finiteness of chiral anomalies and their cancellation in SM.......................................... 10

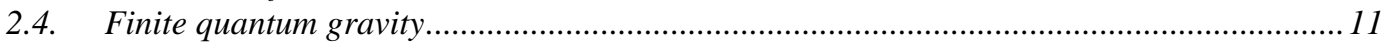

3. Evidence and consequences of the finiteness of QFT ..............................................11

3.1. Evidence and consequences of the finiteness of theories of known fields .........................11

3.2. Consequences for models of unification, supersymmetry and strings.............................. 12

3.3. Consequences for composite models of particles........................................................... 13

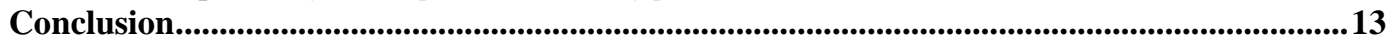

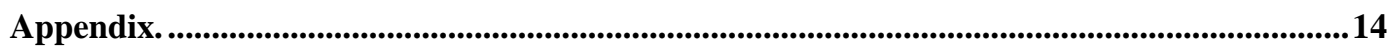

Renormalization constants in QED in the two-loop approximation .................................. 14

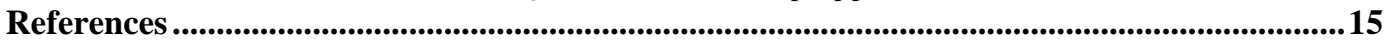

${ }^{1}$ Center for Theoretical Physics and Astrophysics, Tashkent Uzbekistan, zzakir@ qgph.org 


\section{Introduction}

In the first paper [1], it was shown that, at quantization of relativistic fields and strings, the consecutively following to the Stueckelberg-Feynman (SF) treatment leads to a finite theory not containing the zero-point vacuum energy.

In this second paper, interacting fields will be studied and it will be shown that the consistent following to general relativity, taking into account one of its basic effects, gravitational time dilation, leads to a finite theory without ultraviolet divergences.

The ultraviolet divergences in higher orders of perturbation theory (i.e., in the loop diagrams), discovered at the very beginning, still remained the main problem of quantum field theory (QFT). Although there have been successes in its solution in the first two decades, when the weak growth of corrections with cutoff energy $\Lambda$ and the ability to eliminate regularized divergences by renormalization of the bare Lagrangian were clarified, nevertheless, a physical mechanism making the regularizations finite was not found [ 2,3].

In almost all previous attempts to solve this problem, it was naively believed that among the known phenomena there is no one that can eliminate the divergences. Therefore, very radical hypotheses without any experimental basis have been widely promoted for decades, such as supersymmetry, additional dimensions, strings, branes, loops, etc.

In the paper it will be shown that in fact, a physical phenomenon on the basis of the regularization mechanism is the gravitational time dilation which leads to the redshift of frequencies of quanta and the delay of all processes in a strong gravitational field. This is a key phenomenon of general relativity, confirmed by experiments in the 1970s., and it does not exist in the Newtonian theory.

However, in the literature it was usually replaced by one of its manifestations - by the gravitational redshift. The latter exists in the Newtonian theory too, but this former explanation appeared erroneous, and its agreement with the Einstein's prediction in the first order was accidental [4]. Due to such a misunderstanding, this type of time dilation has remained unclear, and its consequences for particle physics and astrophysics have been underestimated. In particular, at leaving the strong gravity region, photon's energy decreases not along its path, as in the Newtonian theory, but the photon is born with a lower frequency due to the gravitational dilation of the local proper times of atoms w.r.t. the world time $t$, marking simultaneous events, and then the photon's energy in terms $t$ conserves.

Standard QFT was based on two prescriptions - to ignore the gravitational effects of high-energy quantum fluctuations, and then to ignore the diverging contributions of these fluctuations to the observables. In the paper it is shown that these two prescriptions are mutually related and the rejection of the first of them, required by general relativity, makes the second one unnecessary.

In a two-particle system with the energy of each particle $h v$, the wavelength $\lambda=c / v$ is inversely proportional to the frequency $v$, and the gravitational radius of the system $r_{g}=2 G \cdot 2 h v / c^{4}$ is proportional $v$ ( $G$ - the gravitational constant). These two curves intersect at $r_{g} \lambda_{c}=\left(2 l_{g}\right)^{2}$, where $l_{g}=\left(G h / c^{3}\right)^{1 / 2}$ is the Planck length. Nearby $r_{g}$, the particles freeze for external observers and their frequencies in terms of $t$ appear as extremely redshifted. Therefore, all fluctuations of the order of Planck energy $\Lambda_{g}=\left(h / G c^{3}\right)^{1 / 2}$ freeze and will not occur in finite world time $t<\infty$, and higher energy processes simply do not occur and do not contribute to the amplitudes of processes [5]. 
Thus, gravitational self-regularization at $\Lambda_{g}$ makes the theory of interacting fields finite. The loop contributions in the theory of gauge fields depend on $\Lambda$ logarithmically and the corrections are small up to $\Lambda_{g}$. Since the effective coupling constant in quantum gravity is also small up to $\Lambda_{g}$, the perturbation theory series converge in this case also.

Thus, the quantum theory of known fundamental fields at quantization on the basis of SF-treatment and the gravitational self-regularization is finite. In this case, the nonlinearity of fields makes stronger the gravitational effects, which lead to even more rapid freezing of processes and suppression of the high energies contributions. The finiteness of the loop corrections makes softer the criterion for the relevance of the models, and now it is sufficient the smallness of these corrections, while renormalizability is only desirable.

In the paper the consequences of the ultraviolet finiteness of the Standard Model and quantum gravity, as well as the prospects for their generalizations are briefly discussed. A more detailed discussion will be presented in the book [6].

In Section 1 the physical foundations of the gravitational regularization in QFT are discussed. In Section 2 the finiteness of the Standard Model and quantum gravity is shown. In Section 3 the evidence and consequences of the finiteness of QFT, as well as further perspectives are discussed.

\section{Gravitational self-regularization of quantized fields}

\subsection{Gravitational freezing of fluctuations at Planck distances}

The theory of relativity introduced into physics three types of relativistic time dilation - inertial, non-inertial, and gravitational. They have significant differences which were usually ignored and this led to a misunderstanding of many phenomena and mistakes in some important applications of general relativity.

The proper time delay of a clock resting in an inertial frame (inertial time dilation) is relative and symmetrical for two inertial frames, as it is shown by the experiment with two light clocks. At the same time, the proper time dilation of clocks resting in a noninertial frame (non-inertial time dilation) is absolute and asymmetrical for two clocks subjected to different accelerations, which is also well known from the clock paradox. Gravitational time dilation, in accordance with the equivalence principle, is similar to the non-inertial one and also asymmetrical and absolute. Below we will study the consequences for the of quantum fields theory of the gravitational time dilation near $l_{g}$.

In QFT, fundamental particles are localized objects, the structure of which can be neglected in processes at the experimentally available energies. However, for the intermediate states the assumption about point particles is no longer justified. Nevertheless, due to problems with nonlocal objects in relativistic theories, these particles also can be considered approximately point-like, as we will assume further.

The interaction vertices of these point particles should also be considered as "pointlike" in order to avoid non-locality again, as for the particles. However, for processes near $l_{g}$ it is difficult to neglect the structure of particles at the interaction vertices, since at these distances the effective "size" of particles, their gravitational radius, also of order $l_{g}$.

Nevertheless, in one a particular case, even under these conditions, the vertices can be considered point-like without internal contradictions in the theory - this is the interaction 
of a quantum particle with classical external fields, in particular, with the gravitational field. Although in this case the interaction can also be represented by the "longitudinal" quanta exchanging diagram, nevertheless, from the physical point of view, here we deal with by the particle's motion in a classical external field. If we know the exact or approximate solution for this field, then the particle trajectories can be searched on this background.

The simplest example is the Coulomb field, and a more complicated case is the Schwarzschild field. The latter case is realized at $l_{g}$ in the loop diagrams, where two or more particles exist simultaneously. In the center of inertia of the particles and at $r>r_{g}$, the external gravitational field of the system can be approximated by the Schwarzschild field, where the components of the metric are: $g_{00}(r)=-g_{11}^{-1}(r)=1-r_{g} / r$.

This metric leads to the redshift of frequencies associated with the physical energy of quanta:

$$
\bar{E}_{\mathbf{p}}=g_{00}^{1 / 2}(r) E_{\mathbf{p}, r}
$$

and $\bar{E}_{\mathbf{p}}$ appears as the source of the external gravitational field of the particles in the loop diagram. For the one-loop diagram, the metric components in the center of inertia of two particle system are determined by $\bar{E}_{\mathbf{p}}$ and take the form:

$$
g_{00}(r)=-g_{11}^{-1}(r)=1-2 G \cdot 2 \bar{E}_{\mathbf{p}} / r c^{4} .
$$

The field operators and their modes are defined on the global hypersurface of simultaneity of distant observers $t=$ const.

The energy $\Lambda_{g}$ and length $l_{g}$ are determined by the conditions that the gravitational radius of the two particles system $r_{g}=2 G \cdot 2 \bar{E}_{\mathbf{p}} / c^{4}$ is of the order of the wavelength of quantum fluctuations of these particles $h c / \bar{E}_{\mathbf{p}}$ and the total energy of the system $2 \bar{E}_{\mathbf{p}}$ is of order $\Lambda_{g}$ :

$$
\frac{2 G \cdot 2 \bar{E}_{\mathbf{p}}}{c^{4}} \simeq \frac{h c}{\bar{E}_{\mathbf{p}}}, \quad 2 \bar{E}_{\mathbf{p}} \simeq\left(h c^{5} / G\right)=\Lambda_{g}=h v_{g},
$$

where $v_{g}=\left(c^{5} / G h\right)$ is the Planck frequency.

Since the gravitational redshift of the frequencies (associated with $\bar{E}_{\mathbf{p}}$ ) is small already at $\sim 3 l_{g}$, at larger distances $r \geq 3 l_{g}$ and lower energies $\bar{E}_{\mathbf{p}} \leq \Lambda_{g} / 3$ we can use the standard formalism of QFT in flat space-time. Due to the strong redshift of $\bar{E}_{\mathbf{p}}$, the contribution to the amplitudes of fluctuation in the interval $l_{g} \leq r \leq 3 l_{g}$ is small and gives only a small correction to the contribution of the main region $3 l_{g} \leq r \leq \infty$.

Indeed, the localization region of a quantum is related to its wavelength as: $r \geq 2 \lambda=2 c / v$. The gravitational radius is proportional to the energy of a two particle system, which is of order $\sim 2 v$ in terms of the coordinate frequency and therefore [5]:

$$
v=v_{0}\left(1-r_{g} / r\right)^{1 / 2} \simeq v_{0}\left(1-\frac{4 G h v}{c^{4}} \frac{v}{c}\right)^{1 / 2}=v_{0}\left(1-v^{2} / \bar{v}_{g}^{2}\right)^{1 / 2},
$$


where $\bar{v}_{g}=v_{g} / 2$, and $v_{g}=\left(c^{5} / G h\right)$ is the Planck frequency. It relates $v_{0}$ by $v$ as:

$$
v_{0} \simeq \frac{v}{\left(1-v^{2} / \bar{v}_{g}^{2}\right)^{1 / 2}}, \quad v \simeq \frac{v_{0}}{\left(1+v_{0}^{2} / \bar{v}_{g}^{2}\right)^{1 / 2}}
$$

It can be seen that even at a very large proper frequency $v_{0} \gg \bar{v}_{g}$, the observed frequencies remain less than half of the Planck frequency: $v<\bar{v}_{g}$, which expresses the freezing of quantum fluctuations in the region $l_{g}$.

In loop diagrams, integration over $v_{0}$ with an infinite limit can be replaced, using (6), by integration over $v$ with the upper limit $v_{g}$. In this case, the differentials are replaced as:

$$
d\left(v_{0}^{2}\right) \simeq \frac{d\left(v^{2}\right)}{\left(1-v^{2} / \bar{v}_{g}^{2}\right)^{2}}, \quad d\left(v^{2}\right) \simeq \frac{d\left(v_{0}^{2}\right)}{\left(1+v_{0}^{2} / \bar{v}_{g}^{2}\right)^{2}} .
$$

In the second case (6), the integrals on $v_{0}$ explicitly converge due to the damping factor $v_{0}^{-4}$; moreover, at $v_{0} \gg \bar{v}_{g}$ all the $v$ in the integrals become constants $v \rightarrow \bar{v}_{g}$.

Note that the gravitational regularization was previously used implicitly as the Pauli-Villars regularization, when particle propagators were replaced by:

$$
\frac{1}{k^{2}-m^{2}} \rightarrow \frac{1}{k^{2}-m^{2}}-\frac{1}{k^{2}-M^{2}}=\frac{1}{k^{2}-m^{2}} \cdot \frac{1}{1-k^{2} / M^{2}} .
$$

Indeed, in the one-loop diagram, the replacement of both propagators according to (7) introduces a factor $k^{-4}$ under the integral, which is equivalent to replacing the differential as in (6).

\subsection{Gravitational self-regularization of loop diagrams}

There have been many attempts in QFT to justify the regularization of the loop diagrams at $l_{g}$ or $\Lambda_{g}$. However, there was no clarity in the underlying physical mechanism and such uncertainty made this regularization as arbitrary as any other one.

In reality, as it is estimated in the previous section, general relativity gives such a physical mechanism for stopping the growth of frequencies at $\Lambda_{g}$, which is no less fundamental than quantum fluctuations and this mechanism is the gravitational time dilation. In the loop diagram, there is an external gravitational field of quanta, which slows down the proper times of quanta w.r.t. the time of the center of inertia and this appears as the gravitational redshift of proper frequencies.

To take into account this relativistic effect, let us consider the system of quanta at $l_{g}$ and the upper limit of the contribution of the gravitational time dilation effects. At quantization of the fields of matter, the action function must include the external gravitational field of high energy fluctuations in the loop diagrams. This external field with the metric $g_{\mu \nu}$ enters into the action function as:

$$
S=\int d^{4} x g^{1 / 2}\left(-\kappa^{-1} R+L_{m}\right),
$$


where $R$ is the Ricci tensor, $g$ is the determinant of the metrics, $\kappa=8 \pi G / c^{4}, L_{m}$ is the Lagrangian of particles and matter fields. The energy-momentum tensor $T_{0}^{0}$, which appears in the corresponding Einstein equations, includes the contributions of not only the bare Lagrangian $L_{m}$, but also the contribution of the perturbation theory series, including the terms renormalizing $L_{m}$.

Next, we need to determine what exactly contributes to $T_{0}^{0}$ in the intermediate states. In the covariant perturbation theory, the energy of an intermediate-state particle is formally associated with the component $p_{0}$ of 4-momentum $p_{\mu}=\left(p_{0}, \mathbf{p}\right)$, where $p_{0}$ describes the invariance under time translations, i.e. not fluctuates, unlike the fluctuating physical energy of quanta $E_{\mathbf{p}}=\left(\mathbf{p}^{2}+m_{0}^{2}\right)^{1 / 2}$. The sum $p_{0}=p_{0(1)}+p_{0(2)}$ for the particles in the one-loop diagram is conserved and therefore it is limited by $p_{0}$ of the initial state, which is many orders of magnitude less than $\Lambda_{g}$.

These distinctions between the two definitions of energy, conserved $p_{0}$ and fluctuating $E_{\mathbf{p}}$, were one of the misleading reasons why gravity effects were not taken into account in the loop diagrams. There was an illusion that $p_{0}$ is energy, while the physical energy entering the right side of Einstein's equations and generating a gravitational field is $E_{\mathbf{p}}$. The contribution to the physical energy of the two particle system in the one-loop diagram is given by $\sim 2 E_{\mathbf{p}}$.

Notice that the $p_{0}$ not only violates the relativistic relationship between energy and momentum, but it also does not have two other basic properties of energy - it is not positive definite (at forward in time evolution) and is not a source of the gravitational field. At the same time, $E_{\mathrm{p}}$ is positive-definite and is a source of the gravitational field ( $\left.T_{0}^{0} \Delta V \sim E_{\mathbf{p}}\right)$, i.e. has all the required for energy properties, with exception the conservation (only the total 3-momentum is conserved at the vertices).

So, at any moment of time $t$, marking simultaneous events in the center of inertia of the loop diagram with $n$ virtual quanta, the total physical energy of order $\Lambda_{g}$ at least of two particles $2 \bar{E}_{\mathbf{p}}$ in the diagram creates an external gravitational field, which can be approximated by the Schwarzschild metric. Other metrics containing the contributions of charge and spin only slightly modify this main contribution and therefore for the estimation of a minimal contribution it is sufficient to take into account this metric only.

But taking into account the strong gravitational effects complicates the theory so much that it is difficult to obtain exact solutions even in simple cases. At the same time, to prove the finiteness and, moreover, the smallness of the loop corrections at such regularization, it's enough for us to get upper limits for these corrections.

If these upper limits give sufficiently small values for loop integrals, then with any further refinement the corrections will decrease only, which will make the situation even more favorable for proving the convergence of the perturbation theory series. Concerning the difference between the "true" values of the corrections and their upper estimate, it will be sufficiently small and will be effectively included in the values of the bare masses and charges, defined as the difference between the physical values of the masses and charges 
and corrections to them. Further refinements therefore will lead only to a small redistribution of the values of the bare parameters and corrections to them.

Let's formulate this procedure for obtaining upper limits in more detail. A strong redshift in the gravitational field of the particles in the loop diagram begins to decrease the energies of quanta at distances of several $l_{g}$, for example, $3 l_{g}$. But a decrease in the fluctuation energy effectively reduces the magnitude of these corrections. Therefore, if we continue to use the dependence on the cutoff energy up to $\Lambda \sim \Lambda_{g}$ without gravitational effects, then the values of the corrections in the interval $3 l_{g} \geq r \geq l_{g}$ will be larger (in absolute value) than their "true" values with the gravitational effects.

This means that a simple cutoff at $\Lambda=\Lambda_{g}$ of the expressions, known from the standard formulation without gravitational effects, gives us an upper limit for the "true" corrections with the gravitational effects. If the values of these upper limits already provide the convergence of the perturbation theory series, then the convergence in a more realistic case, i.e. at accounting the gravitational effects, will be practically proved.

Consider the simplest one-loop diagrams with scalar particles in $\varphi^{4}$ theory. The integral in the correction to the square of mass in the diagram of Fig. 1a depends on the cutoff energy as $\sim \Lambda^{2} / m^{2}$, while in the correction to the vertex in the diagram Figlb is logarithmic $a \ln \left(\Lambda^{2} / m^{2}\right)$.

The contribution to the mass of the power-law correction is very large, of the order $\Lambda \sim \Lambda_{g}$, which makes the theory of scalar fields practically inacceptable. Therefore, scalar particles cannot be fundamental, since they cannot be considered as "point-like" particles up to $l_{g}$. But they can be composite and then their structure should manifest itself at $l \gg l_{g}$ and $E_{p} \ll \Lambda_{g}$.

More interesting is the logarithmic dependence $a \ln \left(\Lambda^{2} / m^{2}\right)$, which takes place for diagrams with gauge fields. As a condition for the convergence of the perturbation theory series under the gravitational regularization at $\Lambda \sim \Lambda_{g}$, we obtain an upper bound for the coefficient $a$ :

$$
a \ln \frac{\Lambda_{g}^{2}}{m^{2}}<1, \frac{1}{a}>\ln \frac{\Lambda_{g}^{2}}{m^{2}} .
$$

This condition can be fulfilled in three cases: either when it is very small, or the mass of the particle is very large, or both at the same time, but so that Eq. (9) holds.

Thus, the external gravitational field of the loop diagrams limits the frequency for the external observer by $\Lambda_{g} / \hbar$ since as higher the proper frequency of quanta, as stronger they freeze in their gravitational field.

\section{Finite Standard Model and Quantum Gravity}

\subsection{The ultraviolet-finite quantum electrodynamics}

The fact that the gravitational self-regularization at $\Lambda_{g}$ makes QFT finite is the first good news. However, in order to be realistic, interactions should yield mass corrections much smaller than $\Lambda_{g}$. This is the case in gauge field theories, where the loop corrections 
are logarithmic and sufficiently small up to $\Lambda_{g}$, and the condition (9) is satisfied at least near $\Lambda_{g}$.

In quantum electrodynamics (QED), the condition (9) is not only satisfied, but also the coefficients $a$ are very small: $a \sim 10^{-3}$. Substitution of the electron mass into the logarithmic factor gives: $\ln \left(\Lambda_{g} / m_{e}^{2}\right) \simeq 103 \sim 10^{2}$ and $a \ln \left(\Lambda_{g} / m_{e}^{2}\right) \sim 10^{-1}$, which shows rather fast convergence of the perturbation theory series.

The initial Lagrangian of spinor QED with "bare" fields and constants (by indices 0 ) has the form:

$$
L_{0}=\bar{\psi}_{0}\left(i \not \partial-m_{0}\right) \psi_{0}-\frac{1}{4}\left(F_{0}^{i k}\right)^{2}-e_{0} \bar{\psi}_{0} \gamma_{i} \psi_{0} A_{0}^{i} .
$$

In QED, as in the renormalizable theory, loop diagrams lead to corrections for each term of the Lagrangian, which depend on the cutoff energy [2,3]:

$$
\delta L=\bar{\psi}_{0}(i \not \partial-\delta m) \psi_{0}\left(Z_{2}-1\right)-\frac{1}{4}\left(F_{0}^{i k}\right)^{2}\left(Z_{3}-1\right)-e_{0} \bar{\psi}_{0} \gamma_{i} \psi_{0} A_{0}^{i}\left(Z_{1}-1\right) .
$$

Thus, the complete Lagrangian, as the sum of (10) and (11), takes the form:

$$
\begin{aligned}
L & =L_{0}+\delta L=Z_{2} \bar{\psi}_{0}\left(i \not \supset-m_{0}-\delta m\right) \psi_{0}- \\
& -\frac{1}{4} Z_{3}\left(F_{0}^{i k}\right)^{2}-Z_{1} Z_{2}^{-1} Z_{3}^{-1 / 2} e_{0}\left(Z_{2} \bar{\psi}_{0} \gamma_{i} \psi_{0} \cdot Z_{3}^{1 / 2} A_{0}^{i}\right) .
\end{aligned}
$$

By introducing the renormalized fields, masses and charges in the form:

$$
\psi=Z_{2}^{1 / 2} \psi_{0}, \quad A^{i}=Z_{3}^{1 / 2} A_{0}^{i}, \quad m=m_{0}+\delta m, \quad e=e_{0} Z_{1}^{-1} Z_{2} Z_{3}^{1 / 2},
$$

we arrive at the complete Lagrangian with renormalized fields and constants:

$$
L=\bar{\psi}(i \not \supset-m) \psi-\frac{1}{4}\left(F^{i k}\right)^{2}-e \bar{\psi} \gamma_{i} \psi A^{i} .
$$

The physical meaning of the four constants $Z_{1}, Z_{2}, Z_{3}$ and $\delta m$ is clear from (12) and (14), and they can be associate with different types of diagrams. As it follows from the definitions of the field propagators, $Z_{2}$ and $Z_{3}$ are the renormalization constants of the electron $\left(S_{c}\right)$ and photon $\left(G_{c}\right)$ propagators, respectively:

$$
\begin{array}{ll}
\left\langle\psi\left(x^{\prime}\right) \bar{\psi}(x)\right\rangle_{0}=Z_{2}\left\langle\psi_{0}\left(x^{\prime}\right) \bar{\psi}_{0}(x)\right\rangle_{0}, & S_{c}=Z_{2} S_{c}^{(0)}, \\
\left\langle A^{i}\left(x^{\prime}\right) A^{k}(x)\right\rangle_{0}=Z_{3}\left\langle A_{0}^{i}\left(x^{\prime}\right) A_{0}^{k}(x)\right\rangle_{0}, & G_{c}=Z_{3} G_{c}^{(0)} .
\end{array}
$$

The renormalized propagators are expressed through the self-energy diagram. In the momentum representation for a photon, the latter is $\Pi\left(q^{2}\right)$, the transverse part of the polarization operator $\Pi_{i k}\left(q^{2}\right)=\left(q_{i} q_{k}-q^{2} g_{i k}\right) \Pi\left(q^{2}\right)$. The correction to the electron mass is separated from the self-energy part $\Sigma(p)$ as:

$$
S_{c}^{-1}=\left(S_{c}^{(0)}\right)^{-1}-\Sigma, \quad \Sigma(p)=\left[\delta m-\left(Z_{2}^{-1}-1\right)+C(p)\right](p-m) .
$$

Then, from the Ward identities:

$$
\frac{\partial \Sigma(p)}{\partial p^{i}}=-\Lambda_{i}(p, p)
$$


where $\Lambda_{i}\left(p, p^{\prime}\right)$ is the contribution of the vertex part, it follows that $Z_{1}=Z_{2}$. Substituting this into (13) gives for the charge: $e=e_{0} Z_{1}^{-1} Z_{2} Z_{3}^{1 / 2}=e_{0} Z_{3}^{1 / 2}$.

In the QED with electrons and photons, the largest one-loop contribution to the renormalization constants gives the diagrams with electrons in the intermediate state. At the following numerical values of main constants and their combinations:

$$
\begin{aligned}
& \alpha=\frac{e_{0}^{2}}{4 \pi} \simeq \frac{1}{137.036} \simeq 0.0073, \quad \frac{\alpha}{4 \pi} \simeq 0.00058, \\
& m_{e} \simeq 0.511 \mathrm{MeV}, \quad \Lambda_{g} \simeq 1.221 \times 10^{19} \mathrm{GeV}, \quad \ln \frac{\Lambda_{g}^{2}}{m_{e}^{2}} \simeq 103.056,
\end{aligned}
$$

the logarithmic factor is equal to:

$$
A_{e}^{(1)}=\frac{\alpha}{4 \pi} \ln \frac{\Lambda_{g}^{2}}{m_{e}^{2}} \simeq 0.0598 .
$$

The contribution of the electron-positron loop to the polarization operator $\Pi\left(q^{2}\right)$ in the limit $q^{2} \rightarrow 0$ is about $8 \%$ :

$$
\Pi(0) \simeq \frac{4}{3} A_{e}^{(1)} \simeq 0.0798 \simeq 7.98 \% .
$$

The renormalization constant of the photon propagator is then equal to:

$$
Z_{3} \simeq 1-\Pi(0)=0.92,
$$

which gives the bare value of the coupling constant and an correction to it about $7.4 \%$ :

$$
\begin{gathered}
\alpha=Z_{3} \alpha_{0}, \quad \alpha_{0} \simeq \frac{\alpha}{0.92} \simeq \frac{1}{126.1}, \\
\delta e^{2}=\Pi(0) e^{2}=0.0798 e^{2} .
\end{gathered}
$$

The renormalization constants of the propagator $Z_{2}$ and the vertex $Z_{1}$ are equal:

$$
Z_{1}^{(1)}=Z_{2}^{(1)} \simeq\left(1+A_{e}^{(1)}\right)^{-1} \simeq 0.944 .
$$

For an electron, the bare mass $m_{e(0)}$ is about $85 \%$ of the physical mass, and the difference between them is about $15 \%$ :

$$
\begin{gathered}
m_{e(0)}=m_{e}\left(1+3 A_{e}^{(1)}\right)^{-1} \simeq 0.8478 m_{e}=0.4332 \mathrm{MэB} \\
\delta m_{e} \simeq 3 A_{e}^{(1)}=0.1795 m_{e(0)}=0.0778 \mathrm{MэB},
\end{gathered}
$$

The values of corrections and bare constants for charged particles of the Standard Model can be calculated similarly.

Other QED loop diagrams, including multi-loop ones, also remain small, and in all cases the gravitational self-regularization leads to the sufficiently small loop contributions to the observables. As an example, in Appendix 2 the two-loop corrections are presented. They are calculated by using the dimensional regularization method, where instead of the logarithmic factor appears $1 / \varepsilon \simeq \ln \left(\Lambda_{g}^{2} / m_{e}^{2}\right)$, This shows that the perturbation theory series in QED are convergent. 


\subsection{Finiteness of other fields of the Standard Model}

In the Standard Model (SM) of particle physics, based on the symmetry group $S U(3) \times S U(2) \times U(1)$ and spontaneous symmetry breaking with a doublet of complex scalar fields [2,3], all loop diagrams are finite due to cutting at $\Lambda_{g}$, for gauge fields they are also small due to the logarithmic dependence on $\Lambda_{g}$ and the asymptotic freedom of two non-Abelian gauge fields.

In electroweak theory and quantum chromodynamics, loop corrections contain a larger number of diagrams than in QED, but even in this case, the contributions practically reduce to modifications of the effective constants in the logarithmic factors (9). At ordinary distances, the coupling constants are larger $\alpha$, but they decrease with decreasing distances and at $\Lambda_{g}$ become smaller $\alpha$. As a result, the perturbation theory with the small distance corrections converges almost as fast as in QED.

In SM, problems arise only in the sector of scalar fields introduced for the spontaneous symmetry breaking. The loop integrals for the scalar fields contain contributions with a power-law growth $\Lambda_{g}^{n}$, which lead to a finite but unacceptably large contribution to the observables. This means that either the scalar particle is composite, and its field is effective, or there is a different mechanism for the generation of masses of SM particles compatible with known facts.

Thus, the SM without zero-point vacuum energy and with gravitational regularization at $\Lambda_{g}$ becomes finite and consistent, since the predicted values of observables are finite and are in accordance with the observations, although taking into account the last remark about the scalar field, which should be considered as a prediction of SM about the nature of this field.

\subsection{Finiteness of chiral anomalies and their cancellation in SM}

A special class of loop diagrams consists the triangular diagrams with fermion propagators only, which grow linearly with cutoff energy $\Lambda$ and lead to the chiral anomalies [3].

At the gravitational regularization with cutting of energies at $\Lambda_{g}$, the contribution of such diagrams gives an expression not with a logarithmic dependence, as in the loops diagrams with gauge bosons, but with a linear dependence on $\Lambda_{g}$. As a result, such diagrams do not diverge, as previously thought, but are finite.

Nevertheless, even being finite, the contribution of the anomalous diagrams to the observables too large that become inadmissible. Therefore, the criterion for the consistency of particle physics models is the mutual cancellation of anomalies in the set of diagrams for each observed process.

In SM, there appears precisely such a cancellation in chiral anomalies and this fact was one of the main selection criteria for both the quark model and models of gauge fields [3].

Notice that the chiral anomaly in the phenomenological models involving $\pi$ mesons, consisting of a quark and an antiquark pair, does not relate to fundamental fields 
and particles, since the introduced local fields and currents of pion are effective and cannot be used at small distances.

Thus, the gravitational regularization at $\Lambda_{g}$ makes the situation with chiral anomalies in theories of gauge fields methodologically reasonable, since here the theory also operates with finite, although very large quantities which ultimately cancel.

\subsection{Finite quantum gravity}

The regularization of loop contributions by their external gravitational field with cutting at $\Lambda_{g}$ occurs in quantum gravity too, since the gravitational time dilation is only amplified by the quantum effects of gravity.

The dimensional coupling constant in quantum gravity leads to power-law contributions from graviton loop diagrams. However, unlike the scalar field, the effective dimensionless coupling constant $G l_{p l}^{2}$ is very small up to $\Lambda_{g}$, so the loop contributions of quantum gravity, both logarithmic and power-law ones, remain small even near $\Lambda_{g}$.

As the result, quantum gravity is not only finite, but it is also possible to apply perturbation theory. Physical observables, therefore, can be represented in the form of bare values and small quantum field corrections. At expanding by these effective coupling constants, the perturbation theory series converges, which will be discussed in more detail in subsequent publications and in the book [6].

\section{Evidence and consequences of the finiteness of QFT}

\subsection{Evidence and consequences of the finiteness of theories of known fields}

If the reformulation of QFT in accordance with the physical requirements of general relativity leads to its ultraviolet finiteness, then there arise questions about which experiments confirm this, whether additional checks are needed, and also what are the consequences of this fundamental fact?

The first evidence of the finiteness of the theories of known fields is the agreement with experiment with high accuracy of the consequences of the SM. This accuracy is mainly limited by the accuracy of the experiments themselves, the accuracy with which physical constants are known, and the accuracy of model approximations in calculating these corrections that can be improved in future.

The most accurate and well tested are the predictions of QED. The agreement with the experiments of QCD and the electroweak theory is also convincing. The smallness of the calculated masses, charges, and magnetic moments of quarks and leptons, where the bare values are determined from a comparison with the observed values, also indicates the smallness of the loop corrections and the convergence of the perturbation theory, which indirectly confirms the gravitational regularization.

Here the question may arise, why does this confirm gravitational, and not some other regularization? The answer is that local QFT is an idealized model based on the assumption about point particles at least up to $l_{g}$. The long searches within its framework led to arbitrary regularizations only, which therefore had to be «removed». On the one hand, the gravitational regularization is required by general relativity and thus cannot be «removed», but on the other hand it appears as the only one providing the ultraviolet finiteness even for «point» particles. 
The consequences of the finiteness of SM and quantum gravity are numerous and non-trivial. Here we note only some of the main ones.

Firstly, a very satisfactory fact is that QFT became finite and consistent in adapting to the already well-known and well-confirmed principles of physics, the requirements of which should have been taken into account from the very beginning. Although in the past this was not done for historical reasons, it is important that in the end it turned out to be possible to do, preserving all previous achievements.

Secondly, there is no need to search and test very radical hypotheses and this makes the situation in particle physics simpler and healthier, since there is still no experimental evidence in favor of such hypotheses.

Thirdly, the long initial stage in the formation of QFT, when it was supposed to be internally contradictory and some serious changes were expected in its foundations, which would remove these contradictions, can now be considered as ended.

Fourth, in describing the structure of the vacuum of fields, the physical picture becomes sufficiently simple, since gravitational freezing excludes the former hypotheses about the «boiling» of vacuum or «foam» at the Planck scale.

And finally, fifth, quantum gravity has become not only consistent, but also significantly simpler than expected, since it has been reduced to the one-loop graviton contributions, while higher-order contributions appeared very small and can be neglected.

\subsection{Consequences for models of unification, supersymmetry and strings}

After the first successful unification experience in the electroweak theory, attempts to further unify the SM fields were natural and will remain one of the main directions in the development of particle physics.

Gravitational regularization further simplifies this situation by the prescription that now scalar fields, as supposedly effective, should not be one of the unified fields.

In addition, attempts to unify the three gauge fields into one were based on the hypothesis that at the energies of order $10^{15} \mathrm{GeV}$, the charges of three known gauge fields become equal. In fact, at the gravitational regularization at $\Lambda_{g}$, in the asymptotic behavior of the three charges their curves intersect only in pairs and at three different points the energy scales of which differ by several orders of magnitude. This indicates that, within the framework of the SM, the Grand Unification of its interactions is either excluded or additional facts, unknown at this stage, are needed.

The advantages of supersymmetry were considered the mutual cancellation of zeropoint energies of bosons and fermions, as well as the finiteness of some loop corrections. But for this, the masses of bosons and fermions should be equal, and also superpartners of particles would have to exist. Due to the absence of the zero-point energy of relativistic fields and the smallness of the loop corrections at the gravitational regularization, there is no need for a hypothesis about such symmetry. The distinctions in the masses of the observed particles and the multiplication of the number of fundamental particles due to the need for superpartners make this artificial symmetry also unrealistic, at least at the level of already known particles.

Another significant simplification of the situation in particle physics is the fact that the local QFT in 4-dimensional space-time proved to be self-contained to solve its former problems. Therefore, now to solve these problems there is no need to use hypotheses about nonlocal primary objects, such as strings, branes or loops, as well as hypotheses about the existence of additional dimensions. These hypotheses and the models based on them themselves had a number of internal contradictions and, formally solving some of the problems, introduced a number of other problems, even greater and difficult to solve. 
Therefore, it is satisfactory to clarify the fact that all these models, while remaining interesting from the point of view of mathematical physics, cease to be directly related to fundamental physics.

A special class of models are models based on the topologically nontrivial configurations of fields. Some of them, with a physically correct formulation of the solving problems, will develop further, since any accounting of nonlinear effects, especially such non-trivial ones, will improve the accuracy of calculations of known phenomena, and may also lead to the prediction and discovery of new phenomena.

\subsection{Consequences for composite models of particles}

Composite models of the SM particles represent another advantageous area for further development of particle physics in addition to the unified models of interactions. The success of composite models would significantly reduce the number of fundamental particles, including the quanta of interactions, which would significantly simplify the unification models and restricts the selection of possible candidates to such models.

However, until now there have been no clear signs of the presence of a substructure in quarks and leptons, and even more so in gauge bosons, and the proposed models were not sufficiently consistent and perfect.

The gravitational regularization opens up new perspectives explicitly for composite models. At first, the composite nature of the scalar boson is predicted, and moreover, on the scales of distances which may be available for experiments in the near future. In this regard, the theoretical problems of composite models become the most relevant and the main ones will probably be solved soon.

Thus, closer attention to composite models and a focus on solving their problems will lead to the fact that further progress in particle physics will be associated with a reasonable perspective from the point of view of historical tradition - a decrease in the number of primary particles and simplification of the models describing them, including models unifying their interactions.

\section{Conclusion}

According to general relativity, at the Planck length, the gravitational radius of the quanta of Planck energy, all processes freeze in the asymptotically flat space of external observers, whose world time is used to construct S-matrices of processes. The freezing of quantum fluctuations leads to the extremal redshift of all frequencies of quanta up to practical vanishing. Thus, the external gravitational field of a system of quanta with such energy, in particular in the loop diagram, leads to the gravitational self-regularization of the frequencies of all quanta in the region of the scale $2 l_{g}$.

The nonlinearity of non-Abelian gauge fields and quantum gravity makes gravitational effects even stronger, which means that freezing occurs faster and at larger distances, which even more strongly suppresses the contributions of high energies.

The invariant cutting of the integrals of the loop diagrams at $\Lambda_{g}$ gives upper bounds for corrections of the perturbation theory series, which for the gauge fields of SM and quantum gravity turn out to be more and more decreasing with the number of loops. This means the convergence of the perturbation theory for these fields. Notice, that this conclusion will not change with further refinement of the calculations, since the refinement means an earlier inclusion of the redshift effects than at $\Lambda_{g}$, which will only further reduce the loop contributions. 
Thus, QFT, taking into account the effects of gravitational time dilation, is ultraviolet-finite. Moreover, the theories of non-renormalizable interactions are also ultraviolet-finite, which makes them consistent when these corrections are small at cutting of integrals at $\Lambda_{g}$. One of such cases is quantum gravity.

This means that in reality in particle physics there has never been a problem of ultraviolet divergences. Therefore, there was no need for new hypotheses to solve this problem. Such radical hypotheses as supersymmetry or extended fundamental objects (strings, branes), as well as the introduction of higher dimensions can no longer be justified as a way to solve this problem. The theoretical basis for such hypotheses is no longer valid, and they will be of interest only when there appear new facts that are not yet available.

In contrast, hypotheses about the composite nature of SM particles retain their significance and will actively develop. Moreover, this direction now also turns out to be the most relevant, since the scalar boson of SM must be composite since the scalar field should be effective, and not fundamental.

A more systematic discussion of the history, details and prospects of the finite QFT will be presented in the book [6].

\section{Appendix.}

\section{Renormalization constants in QED in the two-loop approximation}

The two-loop renormalization constants in QED have the form:

$$
\begin{aligned}
& Z_{1}=1+\frac{\alpha}{4 \pi} Z_{1}^{(1)}+\left(\frac{\alpha}{4 \pi}\right)^{2} Z_{1}^{(2)}+O\left(\alpha^{3}\right), \\
& Z_{2}=1+\frac{\alpha}{4 \pi} Z_{2}^{(1)}+\left(\frac{\alpha}{4 \pi}\right)^{2} Z_{2}^{(2)}+O\left(\alpha^{3}\right), \\
& Z_{3}=1+\frac{\alpha}{4 \pi} Z_{3}^{(1)}+\left(\frac{\alpha}{4 \pi}\right)^{2} Z_{3}^{(2)}+O\left(\alpha^{3}\right) .
\end{aligned}
$$

Here, the one-loop $\left(Z_{i}^{(1)}\right)$ and two-loop $\left(Z_{i}^{(2)}\right)$ factors can be calculated using various methods, sometimes more convenient than the simple cutting. Their results mainly coincide, since small differences slightly change the values of the "bare" masses and charges only.

Below, as an example, the results of calculation in the method of dimensional regularization are presented. This method is very formal, but simplifies the calculations and make them more compact than in the physically more justified regularizations.

In this method, instead of the logarithmic factor, it appears the factor $1 / \varepsilon \simeq \ln \left(\Lambda_{g}^{2} / m_{e}^{2}\right)$ and the constants from (27) take the form [7]:

$$
\begin{aligned}
& Z_{1}^{(1)}=-\frac{3}{\varepsilon}-4+O(\alpha), \quad Z_{1}^{(2)}=\frac{155}{12 \varepsilon}+\frac{5}{2 \varepsilon^{2}}+a_{1}^{(2)}+O(\alpha), \\
& Z_{2}^{(1)}=-\frac{3}{\varepsilon}-4+O(\alpha), \quad Z_{2}^{(2)}=\frac{55}{4 \varepsilon}+\frac{9}{2 \varepsilon^{2}}+a_{2}^{(2)}+O(\alpha), \\
& Z_{3}^{(1)}=-\frac{4}{3 \varepsilon}+O(\alpha), \quad Z_{3}^{(2)}=-\frac{2}{\varepsilon}-15+O(\alpha) .
\end{aligned}
$$

Here 


$$
\begin{aligned}
& a_{1}^{(2)}=\frac{1169}{24}+48 \zeta(2) \ln 2-12 \zeta(3)-\frac{87}{2} \zeta(2), \\
& a_{2}^{(2)}=\frac{7685}{72}+\left(96 \ln 2-\frac{211}{2}\right) \zeta(2)-24 \zeta(3),
\end{aligned}
$$

where $\zeta(n)$ - is the Riemann zeta function, and in the particular case $\zeta(2)=\pi^{2} / 6$.

\section{References}

1. Zakir Z. (2020) Quant. and Grav. Phys., 1:001-7128.

2. Bjorken J.D., Drell S.D. (1964) Relat. Quant. Mech.; Relat. Quant. Fields v.1-2, McG.-H.

3. Itzykson C., Zuber J.-B. (1980) Quantum Field Theory, v.1-2, McG.-H.

4. Okun L.B., et al (1999) Phys.-Uspekhi 42(10) 1045; (2000) Am. J. Phys. 68(2) 115.

5. Zakir Z. (2006) Theor. Phys., Astro. Phys \& Cosmol. 1(2) 28.

6. Zakir Z. (2020) Finite Quantum Field Theory, CTPA, T.

7. Hönemann I., et al (2018) Phys. Rev., D 98, 113008. 